\title{
Quantitative Sensitivity Analysis of Surface Attached Optical Fibre Strain Sensor
}

\author{
Kai Tai Wan
}

\begin{abstract}
Optical fibre strain sensors, especially the fibre Bragg grating (FBG) type, are widely applied in different applications. The most common installation method is surface-attached. In principle, optical fibre strain sensor with adequate sampling and signal processing techniques is usually more accurate than electrical resistive strain gauge. However, the strain of the surface of structure may not transfer to the sensing element perfectly. The ratio between the measured and actual strain can be correlated by a strain transfer factor (STF). However, it depends on the material and geometrical properties of the optical fibre and adhesive. It is non-economical and impractical to measure STF for every installed sensor. It is desirable to identify the most sensitive parameters on the variation of STF so that the quality control and assurance procedure can be performed more efficiently. In this paper, a quantitative global sensitivity analysis, called extended Fourier amplitude sensitivity test will be performed to compute the first-order and total sensitivity indexes based on a well-established semi-analytical/empirical mechanical model of three material and five geometrical parameters of both integral and OFBG type optical fibre strain sensor with two different kinds of polymeric coating under three types of strain field in sixteen different configurations. From the detail analysis, the most sensitive parameters on STF are bond length, the thickness of adhesive beneath the optical fibre and the deviation of grating position, which are related to workmanship instead of the material properties of optical fibre and adhesive.
\end{abstract}

Index Terms-optical fibre strain sensor, strain transfer, extended Fourier amplitude sensitivity test, global sensitivity analysis.

\section{INTRODUCTION}

$\mathbf{O}$ PTICAL fibre strain sensor is widely used in many applications. It possesses many advantages over conventional resistive strain gauge. It is small size, intrinsically immune to electromagnetic interference and low signal attenuation in transmission cable. With proper packaging techniques, the sensor is versatile, robust and durable. Optical fibre strain sensor can be divided into two major types, integral and point sensor. Integral strain sensors are mostly interferometric based, such as Mach-Zehnder interferometer [1], Fabry-Perot interferometer [2], [3] and white light interferometer [2], [4]. They measures the interference pattern of two separate optical paths from the same source. The most popular point type sensor is optical fibre Bragg grating (OFBG) strain sensor [5], [6]. There are many methods available to interrogate the Bragg wavelength from OFBG sensors [6], [7]. The most common applications of optical fibre strain sensor are to measure strain for structural health monitoring/evaluation [8]-[10], damage detection of fibre reinforced polymeric composites [11]-[13], fatigue crack monitoring [14] as well as many experimental

K.T. Wan is with Nano and Advanced Materials Institute Limited, Hong Kong, e-mail: ktwan@nami.org.hk. works in structural and material engineering [15], [16]. In addition, OFBG strain sensors are used as the major sensing component for monitoring different physical (force, acceleration, pressure, displacement,temperature, humidity) and chemical ( $\mathrm{pH}$, chemical concentration) quantities [17]-[19].

Optical fibre strain sensors are commonly attached on the surface of structure similar to resistive strain gauges [20], [21]. In principle, with adequate sampling and signal processing techniques, the accuracy of optical fibre strain sensor is better than electrical counterparts. However, from the previous studies [22]-[27], the mechanical strain transfer from the surface of structure to the sensing element of optical fibre strain sensor (the core or grating of the optical fibre) may not be perfect. The effectiveness of strain transfer depends on bond length, sensor position, the material and geometrical properties of the optical fibre as well as the adhesive. In practice, all influential factors are incorporated by a single calibration factor. To improve the reliability, reproducibility and repeatability of surface attached optical fibre strain sensor, the most sensitive parameter(s) on calibration factor should be identified and quantified, hence, the sensor fabrication and installation techniques can be optimised in order to minimise the uncertainties efficiently.

From the previous studies [26]-[28], only local sensitivity analysis was performed. They could not provide quantitative correlation on the calibration factor among those parameters. In this paper, a computationally efficient algorithm is used to quantitatively compute the global sensitivity indexes on the calibration factor from different material and geometrical parameters under different types of strain field. The effects on the strain measurement variation by the uncertainties of optical properties as well as different sampling and peak evaluation techniques will not be in the scope of this paper. Section II briefly reviews the mechanical strain transfer model of surface attached optical fibre strain sensor and the quantitative global sensitivity analysis algorithm called extended Fourier Amplitude Sensitivity Test (eFAST). Section III describes the cases in this study with different fibre coating materials, geometries, strain fields and sensor types. Section IV discusses the results of the sensitivity analysis. The important insights from this study will be summarised in Section V.

\section{Summary of the Mechanical Model and Global Sensitivity Analysis}

In this section, the mechanical model of surface attached optical fibre strain sensor and the extended Fourier Amplitude Sensitivity Test are briefly reviewed for completeness. 


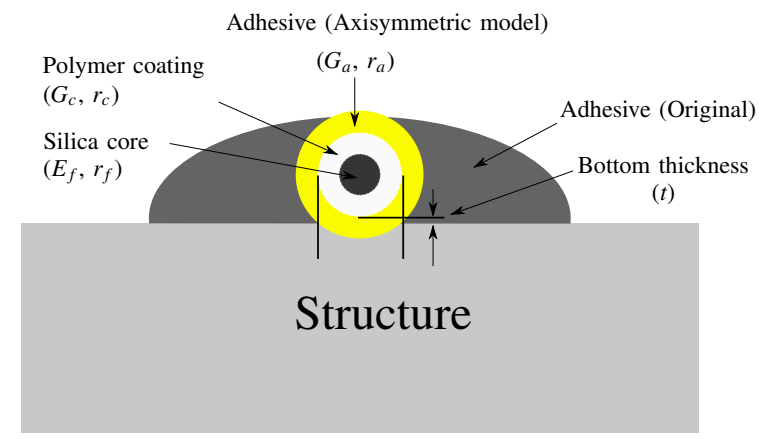

Fig. 1. Typical cross-section of a surface attached fibre optic strain sensor

\section{A. Mechanical model of surface attached optical fibre strain sensor}

Typical cross-section of a surface attached optical fibre strain sensor is shown in Fig. 1. The core and cladding are both made of silica. They possess similar mechanical properties but different in optical properties. In later part of this paper, they are referred collectively as silica core.

The mechanical strain transfer from the surface of structure to the silica core is approximated by an axisymmetric shear lag model [29]. The model assumes (i) all materials are linearly elastic, (ii) all material properties are isotropic and homogeneous, (iii) no debonding at all interfaces, (iv) the silica core carries pure axial stress except at the interface with the polymeric coating, (v) the polymeric coating and adhesive are deformed by shearing and do not carry any axial stress and (vi) the stress distribution is axisymmetric. From these assumptions, the shear stress at any point can be related to the shear stress at the silica core/coating interface.

By considering the force equilibrium of the silica core from the free-body diagram in Fig. 2 and the shear lag model in the polymeric coating and adhesive layer, the relationship between the mechanical strain of the silica core $\left(\varepsilon_{f}\right)$ and the strain of the surface of structure $\left(\varepsilon_{s}\right)$ is given by Equation 1 [26].

$$
\frac{d^{2} \varepsilon_{f}(z)}{d z^{2}}-\alpha_{0}^{2} \varepsilon_{f}(z)=-\alpha_{0}^{2} \varepsilon_{s}(z)
$$

where $\alpha_{0}$ is referred as shear lag characteristic, which incorporates all the material and geometrical properties of the optical fibre and adhesive layer. It is given by Equation 2.

$$
\alpha_{0}^{2}=\frac{2}{k r_{f} E_{f}}
$$

where $r_{f}$ and $E_{f}$ are the radius and elastic modulus of silica core, respectively, and $k$ relates the shear modulus and geometrical properties of the polymeric coating and adhesive layer and it is given in Equation 3.

$$
k=r_{f}\left(\frac{1}{G_{c}} \ln \frac{r_{c}}{r_{f}}+\frac{1}{G_{a}} \ln \frac{r_{c}}{r_{a}}\right)
$$

where $r_{c}$ and $r_{a}$ are the radii of coating and adhesive, respectively and $G_{c}$ and $G_{a}$ are the shear moduli of coating and adhesive, respectively. The radius of the adhesive layer in the axisymmetric model is equal to $\sqrt{\left(r_{c}+t\right)^{2}+r_{c}^{2}}$ where $r_{c}$ is the radius of polymeric coating of the optical fibre and $t$ is the

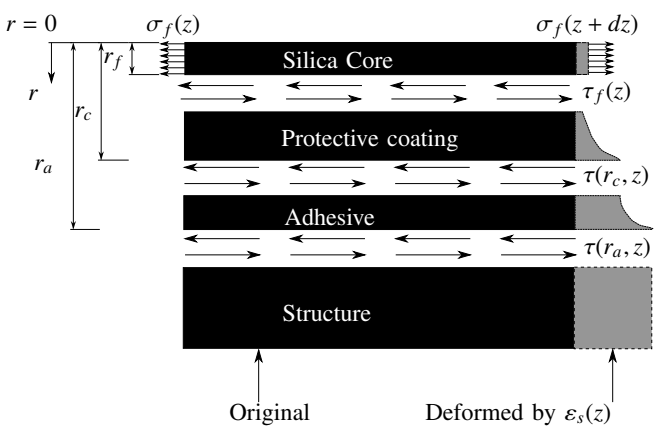

Fig. 2. Free body diagram of the silica core and protective coating

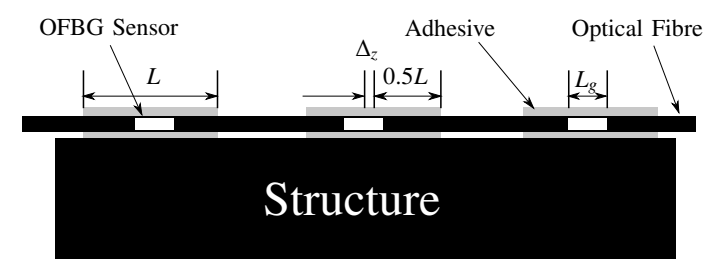

Fig. 3. Typical configuration of surface-attached OFBG strain sensor

thickness of adhesive beneath the optical fibre. The constants of the general solution of Equation 1 can be determined by specifying the boundary conditions that there is no mechanical strain at both ends of the bonded section of the optical fibre, i.e. $\varepsilon_{f}(0)=\varepsilon_{f}(L)=0$.

It was found that the solution of Equation 1 deviates from the results of experiments and finite element model when shear lag characteristic is larger than 0.4 (the units of length and modulus are in $\mathrm{mm}$ and $\mathrm{N} / \mathrm{mm}^{2}$, respectively) [26]. The reason is that the assumptions (v) and (vi) may not be valid when the axial stiffness of the adhesive is non-negligible compared to the silica core. To preserve the elegant form of the mechanical model, [26] suggested modifying the shear lag characteristic semi-empirically by Equation 4 .

$$
\alpha=\left\{\begin{array}{l}
\alpha_{0}, \text { when } \alpha_{0}<0.4 \\
A\left(1-e^{-B \alpha_{0}}\right), \text { otherwise }
\end{array}\right.
$$

where the values $A$ and $B$ are determined by the thickness of adhesive layer underneath the optical fibre ( $t$ in $\mathrm{mm}$ ) and they are given in Equation 5.

$$
\left\{\begin{array}{l}
A=0.48-0.59 \ln t \\
B=1.71 t+0.41
\end{array}\right.
$$

Hence, the shear lag characteristic $\alpha_{0}$ in Equation 1 is replaced by the effective shear lag characteristic $\alpha$.

For OFBG strain sensor, the gauge length (grating) $L_{g}$ is shorter than the bond length $(L)$. The grating may be located at $\Delta_{z}$ from the mid point of the bonded section (Fig. 3). For integral strain sensor (such as interferometer), the gauge length is equal to the bond length and $\Delta_{z}=0$. The calibration factor or more precisely the strain transfer fraction $\beta$ can be computed by Equation 6 , where $\varepsilon_{f}(z)$ can be obtained by solving Equation 1 with given strain of the surface of structure $\varepsilon_{s}(z)$ and appropriate boundary conditions. 


$$
\beta\left(\alpha, \Delta_{z}, L_{g}\right)=\frac{\int_{\frac{L-L_{g}}{2}+\Delta_{z}}^{\frac{L+L_{g}}{2}}+\Delta_{z}}{\int_{\frac{L-L_{g}}{2}}^{\frac{L+L_{g}}{2}} \varepsilon_{S}(z) \mathrm{d} z}
$$

B. Global sensitivity analysis - extended Fourier Amplitude Sensitivity Test

Sensitivity analysis (SA) is a tool to investigate how the model output depends on the input factors. In practice, there are two different schools of philosophy can be identified, local SA [30] and global SA [31]-[33]. For the first one, the local response of the output is investigated by varying one of the input factors while holding the others unchanged at their central (nominal) values. This involves partial derivative with possibly normalisation by the nominal value of the factor or by its standard deviation. The school of global SA tries to explore the domain of the input factors globally within a finite region and examines the variation of the model output by all factors collectively. The classical method is Monte Carlo simulation. However, it explores the domain randomly and hence, the computational efficiency is low. The more efficient methods of global SA are Fourier amplitude sensitivity test (FAST) [34], [35] and its modified version extended FAST (eFAST) [36] that explore the multidimensional space spanned by the input factors from a suitably defined search-curve. It can compute both the first-order and total sensitivity indexes efficiently. The total sensitivity index of the i-th factor $S_{T i}$ is defined as the sum of all sensitivity indexes in all order involving factor $i$ [32]. For example, for a model with three input factors, the total sensitivity index of the second factor on the output variance $S_{T 2}$ is given by Equation 7 .

$$
S_{T 2}=S_{2}+S_{21}+S_{23}+S_{123}
$$

where $S_{2}$ is the first-order sensitivity index for the second factor, $S_{2 j}$ is the second-order sensitivity index for the coupled effects by factor 2 and $j(j \neq 2)$ and $S_{123}$ is the third-order sensitivity index.

The principle of eFAST is that each parameter oscillates with different frequencies $\omega_{i}$. While the value of each input factor $\left(X_{i}\right)$ is normalised to a range between 0 and $1\left(x_{i}\right)$ and then parameterised by a real number $s$. Equation 8 shows a set of searching curves oscillating between 0 and 1 with $s$.

$$
x_{i}(s)=\frac{1}{2}+\frac{1}{\pi} \arcsin \left(\sin \left(\omega_{i} s+\varphi_{i}\right)\right)
$$

where $\varphi_{i}$ is a random phase-shift of the i-th factor chosen uniformly in $[0,2 \pi)$. Consider a model $y=f(\mathbf{x}(s))$ where $y$ is the model output and $\mathbf{x}$ is the vector of the normalised input factors, it can be transformed to $y=g(s)$, which can be expanded in a Fourier series as in Equation 9 and the Fourier coefficients $\left(c_{k}\right)$ can be computed by Equation 10 or numerically by fast Fourier transform algorithm.

$$
\begin{gathered}
y=g(s)=\sum_{k=-\infty}^{\infty}\left(c_{k} e^{i k s}\right) \\
c_{k}=\frac{1}{2 \pi} \int_{-\pi}^{\pi} g(s) e^{-i k s} \mathrm{~d} s
\end{gathered}
$$

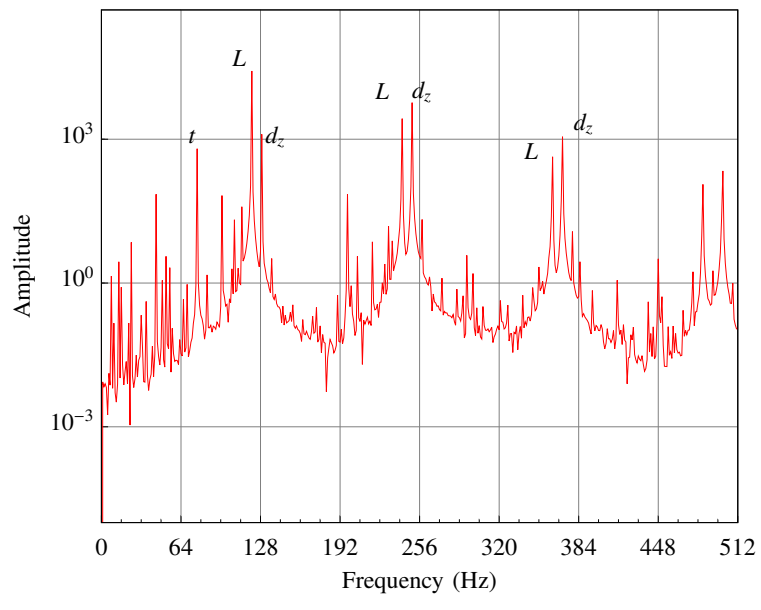

Fig. 4. Frequency spectrum for calculating the first-order sensitivity indexes of all factors at the same time

TABLE I

THE OSCILLATING FREQUENCIES $\left(\omega_{i}\right)$ FOR THE FIRST-ORDER AND TOTAL SENSITIVITY INDEXES

\begin{tabular}{l|cccccccc} 
& $r_{f}$ & $r_{c}$ & $t$ & $E_{f}$ & $G_{c}$ & $G_{a}$ & $L$ & $\Delta_{z}$ \\
\hline For $S_{i}$ & 23 & 55 & 77 & 97 & 107 & 113 & 121 & 125 \\
\hline For $S_{T i}$ & 1 & 17 & 33 & 49 & 1024 & 65 & 81 & 97 \\
\hline
\end{tabular}

To compute the first-order sensitivity index of the i-th factor $\left(S_{i}\right)$, the set of oscillating frequencies is chosen to be linearly independent integers. The oscillating frequencies used in this study for computing the first-order sensitivity indexes are shown in the first row of TABLE I. Fig. 4 shows the frequency spectrum for computing the first-order sensitivity indexes of one of the cases of the sensitivity analysis in the following section. The Fourier coefficients $\left(c_{k}\right)$ are then used to estimate the total variance $(D)$ and the variance of the i-th factor $\left(D_{i}\right)$ of the model output in Equation 11 and 12, respectively. $S_{i}$ can then be computed from Equation 13.

$$
\begin{aligned}
D & =2 \sum_{k=1}^{\infty}\left|c_{k}\right|^{2} \\
D_{i} & =2 \sum_{p=1}^{\infty}\left|c_{p \omega_{i}}\right|^{2} \\
S_{i} & =\frac{D_{i}}{D}
\end{aligned}
$$

The accuracy of the estimation can be improved by resampling scheme by choosing $N_{r}$ different sets of random phase-shift $\left(\varphi_{i 1}, \varphi_{i 2}, \cdots, \varphi_{i_{N_{r}}}\right)$ and then different sets of searching curves can be generated. In the re-sampling scheme, the minimum sample size $N_{s}$ is given by Equation 14 .

$$
N_{s}=N_{r}\left(2 M \omega_{\max }+1\right)
$$

where $\omega_{\max }$ is the maximum oscillating frequency of all input factors and $M$ is an integer $\geq 2$. In this study, $M$ and $N_{r}$ are both set at 4. $D$ and $D_{i}$ are computed independently over each of the random searching curves and their arithmetic means are calculated for the estimation of $S_{i}$ in Equation 13. 


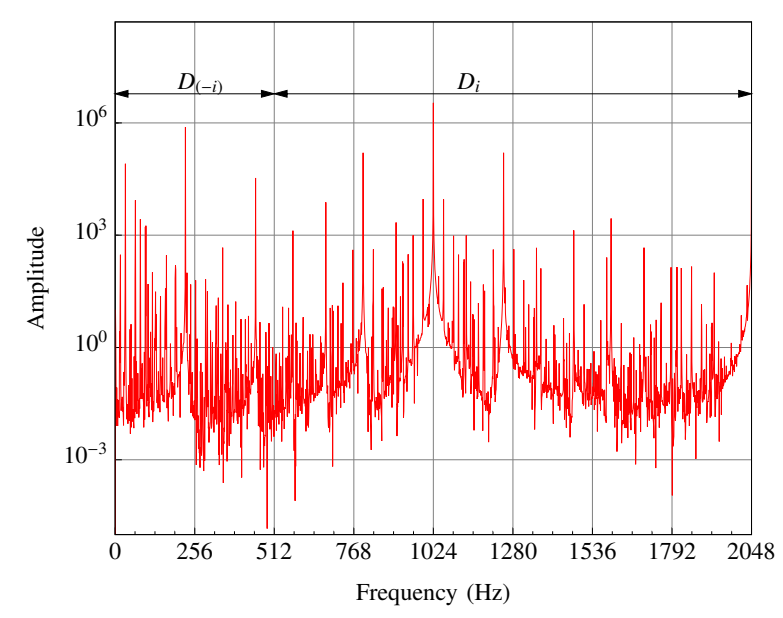

Fig. 5. Frequency spectrum for calculating the total sensitivity index of factor $i$, which oscillates at $1024 \mathrm{~Hz}$

To calculate the total sensitivity index of the i-th factor $\left(S_{T i}\right), \omega_{i}$ is set at several times higher than all other factors. To save the computational effort, $D_{(-i)}$ in Equation 15 is computed and it represents the variation of all factors except the i-th factor. The set of oscillating frequencies adopted in this study and an example of frequency spectrum for total sensitivity indexes computation of the i-th factor are given in the second row of TABLE I and Fig. 5, respectively. From Equation 11 and Equation 15, $S_{T i}$ can be computed by Equation 16. Unlike the first-order sensitivity indexes, which can be computed efficiently by a single set of oscillating frequencies, the total sensitivity indexes must be computed one by one.

$$
\begin{aligned}
D_{(-i)} & =2 \sum_{k=1}^{\omega_{i}-1}\left|c_{k}\right|^{2} \\
S_{T i} & =\frac{D-D_{(-i)}}{D}
\end{aligned}
$$

\section{Details of the Cases for Sensitivity Analysis}

In this section, the details of the sixteen cases for sensitivity analysis in TABLE II will be discussed. They are the combinations of four categories. The first is fibre coating types. There are two common coating materials, acrylate and polyimide, for optical fibre with silica core. The second is bond length. When there is enough space for sensor installation, the bond length is usually at least $30 \mathrm{~mm}$ to allow sufficient strain transfer. When the strain sensor is used as the sensing component which transforms other quantity into strain, due to the space limitation in some situations for sensor miniature and geometrical constraints [10], the bond length may be only $15 \mathrm{~mm}$ or even shorter. Hence, two different bond lengths $15 \mathrm{~mm}$ and $30 \mathrm{~mm}$, are considered. The third category is strain field type. Three different types of strain field are considered. They are (i) uniform, (ii) quadratic and (iii) reciprocal strain fields, which will be further discussed in later part of this section. The last category is the sensor type, integral or grating. For integral sensor, such as interferometer, it measures the integral length change of the whole gauge length and $\Delta_{z}=0$.
TABLE II

LIST OF THE SIMULATED CASES

\begin{tabular}{c|cccc} 
Case & coating & bond length & strain field & sensor \\
\hline 1 & acrylate & $15 \mathrm{~mm}$ & uniform & grating \\
2 & acrylate & $15 \mathrm{~mm}$ & uniform & integral \\
3 & acrylate & $15 \mathrm{~mm}$ & quadratic & grating \\
4 & acrylate & $15 \mathrm{~mm}$ & reciprocal & grating \\
\hline 5 & acrylate & $30 \mathrm{~mm}$ & uniform & grating \\
6 & acrylate & $30 \mathrm{~mm}$ & uniform & integral \\
7 & acrylate & $30 \mathrm{~mm}$ & quadratic & grating \\
8 & acrylate & $30 \mathrm{~mm}$ & reciprocal & grating \\
\hline 9 & polyimide & $15 \mathrm{~mm}$ & uniform & grating \\
10 & polyimide & $15 \mathrm{~mm}$ & uniform & integral \\
11 & polyimide & $15 \mathrm{~mm}$ & quadratic & grating \\
12 & polyimide & $15 \mathrm{~mm}$ & reciprocal & grating \\
\hline 13 & polyimide & $30 \mathrm{~mm}$ & uniform & grating \\
14 & polyimide & $30 \mathrm{~mm}$ & uniform & integral \\
15 & polyimide & $30 \mathrm{~mm}$ & quadratic & grating \\
16 & polyimide & $30 \mathrm{~mm}$ & reciprocal & grating
\end{tabular}

TABLE III

LIST OF MATERIAL AND GEOMETRICAL PROPERTIES

\begin{tabular}{lcc} 
& $\min$ & $\max$ \\
\hline$r_{f}(\mu \mathrm{m})$ & 62.15 & 62.85 \\
$r_{c}(\mu \mathrm{m}):$ acrylate & 117.5 & 130 \\
$r_{c}(\mu \mathrm{m}):$ polyimide & 90 & 105 \\
$t(\mathrm{~mm})$ & 0.05 & 0.5 \\
$E_{f}(\mathrm{GPa})$ & 50 & 90 \\
$G_{c}(\mathrm{GPa}):$ acrylate & 0.2 & 0.3 \\
$G_{c}(\mathrm{GPa}):$ polyimide & 0.9 & 1.5 \\
$G_{a}(\mathrm{GPa})$ & 0.8 & 3.0 \\
$L(\mathrm{~mm}):$ short gauge length & 10 & 20 \\
$L(\mathrm{~mm}):$ long gauge length & 25 & 35 \\
$\Delta_{z}(\mathrm{~mm})$ & -3 & 3
\end{tabular}

For grating type, the strain sensitive region is only the grating part that may deviate from the mid-point of the bond length by $\Delta_{z}$.

\section{A. Material and geometrical properties}

Eight material and geometrical related parameters of the optical fibre as well as adhesive will be considered. They are (i) radius of silica core $\left(r_{f}\right)$, (ii) radius of coating $\left(r_{c}\right)$, (iii) thickness of adhesive beneath the optical fibre ( $t$ ), (iv) elastic modulus of silica core $\left(E_{f}\right),(\mathrm{v})$ shear modulus of coating $\left(G_{c}\right)$, (vi) shear modulus of adhesive $\left(G_{a}\right)$, (vii) bond length $(L)$ and (viii) deviation of the center of the grating from the mid-point of the bond length $\left(\Delta_{z}\right)$ for OFBG strain sensor (see Fig. 1 and 3). TABLE III shows the ranges of those parameters in the global sensitivity analysis. The properties of silica core is referred to SMF28, which is one of the most popularly used optical fibre for strain sensor. The shear modulus of adhesive is referred to cyanoacrylate type while its thickness covers the range of common practical installation methods.

\section{B. Uniform and nonuniform strain field}

Based on interrogation techniques, OFBG strain sensor can measure non-uniform strain field [13], [37]. However, no matter how sophisticated and accurate interrogation techniques are used to recover non-uniform strain field along the grating from the reflective spectrum, it is impossible to recover the strain field of the surface of structure if the sensitive parameters of mechanical strain transfer are not well controlled. In this 


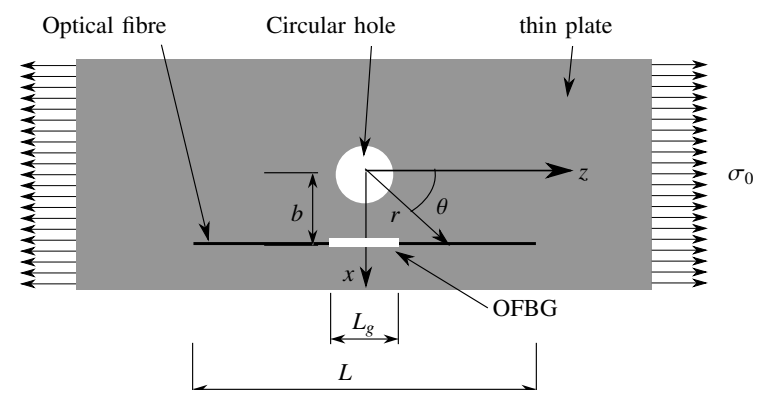

Fig. 6. Thin plate with circular hole under uniform tensile stress

study, the mechanical strain transfer of three different types of strain field are considered. They are uniform, quadratic and reciprocal.

1) Uniform strain field: When the bond length is small compare with the structure and the strain gradient is negligible along the bond length, the strain field can be considered as uniform and assumed to be constant $\left(\varepsilon_{s}(z)=\varepsilon_{s}\right)$. The general solution of Equation 1 under uniform strain field is given by Equation 17.

$$
\varepsilon_{f}(z)=\varepsilon_{s}\left(1+\tanh \frac{\alpha L}{2} \sinh \alpha z-\cosh \alpha z\right)
$$

2) Quadratic strain field: When the strain sensor is attached on the bottom surface of a simply-supported beam under uniformly distributed load and the bond length is comparable with the span, the strain field is quadratic and the strain distribution can be generally represented by $\varepsilon_{s}(z)=c_{1} z^{2}+c_{2} z+c_{3}$, where $c_{1}, c_{2}$ and $c_{3}$ are constants. The solution of Equation 1 under quadratic strain field is given by Equation 18 .

$$
\begin{aligned}
\varepsilon_{f}(z) & =\left(\frac{c_{3} \alpha^{2}+2 c_{1}}{\alpha^{2}} \tanh \frac{\alpha L}{2}-\frac{c_{1} L^{2}+c_{2} L}{\sinh \alpha L}\right) \sinh \alpha z \\
& -\frac{c_{3} \alpha^{2}+2 c_{1}}{\alpha^{2}} \cosh \alpha z+c_{1} z^{2}+c_{2} z+\frac{c_{3} \alpha^{2}+2 c_{1}}{\alpha^{2}}
\end{aligned}
$$

3) Reciprocal strain field: When the strain sensor locates near a circular hole or a crack tip, the strain field is reciprocal. The strain field is highly nonlinear near stress concentration region. In Fig. 6, a circular hole is at the centroid of a thin homogeneous plate that the thickness is small enough so that the plane stress assumption is valid. Assume the radius of the hole is small compared with the width of the rectangular plate and take the origin at the center of the circular hole, the stress state in polar coordinates can be solved analytically as in Equation 19. The stresses in polar coordinates can be transformed to rectangular coordinates by Equation 20. When the optical fibre strain sensor is attached on the plate along the longitudinal direction and with distance $b$ from the center of the circular hole (Fig. 6), the polar coordinates of the optical fibre can be calculated by Equation 21. With considering the Poisson's effect, the longitudinal strain field along the optical fibre is given by Equation 22 .

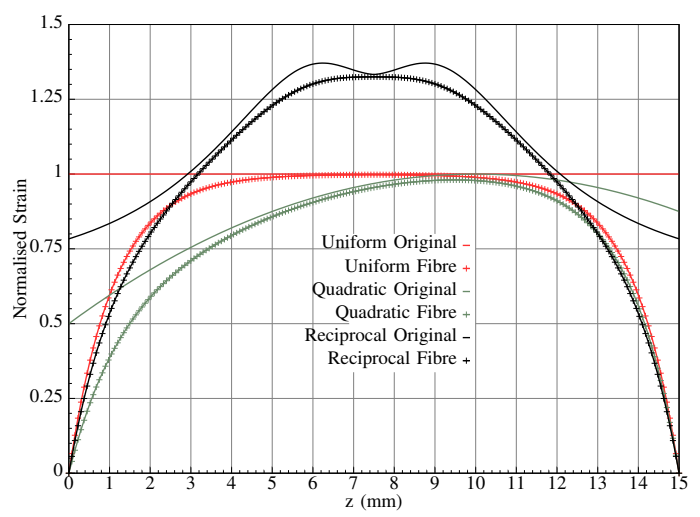

Fig. 7. Examples of strain along the optical fibre of different types of strain field on the surface of structure

\section{TABLE IV}

VALUES OF THE COEFFICIENTS OF DIFFERENT STRAIN FIELDS

\begin{tabular}{lll} 
Uniform strain field & Quadratic strain field & Reciprocal strain field \\
\hline$\varepsilon_{s}=1$ & $c_{1}=0.5$ & $a=50$ \\
& $c_{2}=1$ & $b=55$ \\
& $c_{3}=2$ &
\end{tabular}

$$
\begin{gathered}
\left\{\begin{array}{l}
\sigma_{r}=\frac{\sigma_{0}}{2}\left(1-\frac{a^{2}}{r^{2}}\right)+\frac{\sigma_{0}}{2}\left(1+\frac{3 a^{4}}{r^{4}}-\frac{4 a^{2}}{r^{2}}\right) \sin 2 \theta \\
\sigma_{\theta}=\frac{\sigma_{0}}{2}\left(1+\frac{a^{2}}{r^{2}}\right)-\frac{\sigma_{0}}{2}\left(1+\frac{3 a^{4}}{r^{4}}\right) \cos 2 \theta \\
\tau_{r \theta}=-\frac{\sigma_{0}}{2}\left(1-\frac{3 a^{4}}{r^{4}}+\frac{2 a^{2}}{r^{2}}\right) \sin 2 \theta
\end{array}\right. \\
\begin{cases}\sigma_{z}=\sigma_{r} \cos ^{2} \theta+\sigma_{\theta} \sin ^{2} \theta-2 \tau_{r \theta} \sin \theta \cos \theta \\
\sigma_{x}=\sigma_{r} \sin ^{2} \theta+\sigma_{\theta} \cos ^{2} \theta+2 \tau_{r \theta} \sin \theta \cos \theta \\
\tau_{x z}=\left(\sigma_{r}-\sigma_{\theta}\right) \sin \theta \cos \theta+\tau_{r \theta}\left(\cos ^{2} \theta-\sin ^{2} \theta\right)\end{cases} \\
\left\{\begin{array}{l}
r=\sqrt{z^{2}+b^{2}} \\
\theta=\arctan \frac{b}{z}
\end{array}\right. \\
\varepsilon_{S}(z)=\frac{1}{E}\left(\sigma_{z}-v \sigma_{x}\right)
\end{gathered}
$$

It is difficult to have a close-form solution of Equation 1 with the right hand side as Equation 22. However, it can be solved numerically by finite element method. Fig. 7 shows an example of the strain distribution along the optical fibre under different types of strain field. The values of the coefficients of all three different strain fields are shown in TABLE IV.

\section{Results and Discussion of Numerical Simulations}

Fig. 8 shows the strain transfer fractions $(\beta)$ and the firstorder sensitivity indexes $\left(S_{i}\right)$ of all cases in TABLE II. There are three material and five geometrical parameters investigated in this global sensitivity analysis. The numerical values of the average, minimum and maximum strain transfer fraction during the computation of the sensitivity indexes are shown in TABLE V. According to TABLE VI, the total contribution of the first-order sensitivity indexes by the variations of radius and elastic modulus of silica core, radius and shear modulus 


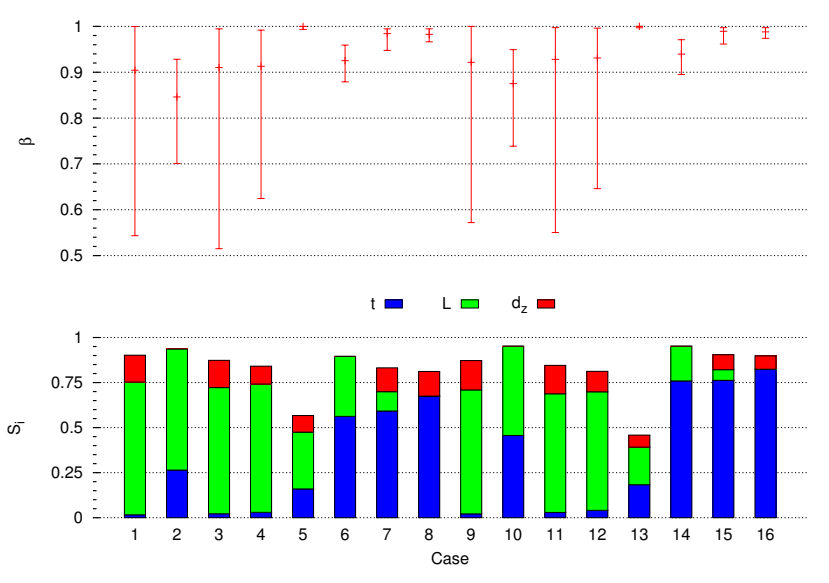

Fig. 8. The effective strain transfer fraction $(\beta)$ and the first-order sensitivity indexes $\left(S_{i}\right)$

TABLE V

STRAIN TRANSFER FRACTIONS OF ALL SIMULATED CASES

\begin{tabular}{lllll} 
Case & Ave. $\beta$ & Min. $\beta$ & Max. $\beta$ & $\Delta \beta$ \\
\hline 1 & 0.904 & 0.543 & 1.000 & 0.457 \\
2 & 0.846 & 0.701 & 0.928 & 0.227 \\
3 & 0.910 & 0.515 & 0.995 & 0.480 \\
4 & 0.913 & 0.625 & 0.992 & 0.367 \\
\hline 5 & 1.000 & 0.993 & 1.000 & 0.007 \\
6 & 0.925 & 0.879 & 0.959 & 0.080 \\
7 & 0.984 & 0.948 & 0.995 & 0.047 \\
8 & 0.983 & 0.966 & 0.995 & 0.028 \\
\hline 9 & 0.922 & 0.572 & 1.000 & 0.428 \\
10 & 0.875 & 0.739 & 0.949 & 0.210 \\
11 & 0.928 & 0.550 & 0.997 & 0.447 \\
12 & 0.931 & 0.646 & 0.996 & 0.350 \\
\hline 13 & 1.000 & 0.997 & 1.000 & 0.003 \\
14 & 0.939 & 0.895 & 0.971 & 0.076 \\
15 & 0.989 & 0.961 & 0.997 & 0.036 \\
16 & 0.988 & 0.974 & 0.997 & 0.023
\end{tabular}

of polymeric coating as well as shear modulus of adhesive is less than 10 percent in all cases and the variations of the strain transfer fraction due to these 5 parameters are less than 1 percent. That means the strain transfer fraction is insensitive to the variation of material and geometrical properties of typical optical fibre strain sensor and the material property of the adhesive beneath the optical fibre. The remaining three sensitive parameters are bond length, thickness of adhesive beneath the optical fibre and the deviation of the mid-point of the grating from the mid point of the bond length. All three parameters are related to workmanship of surface attach optical fibre strain sensor. The first-order and total sensitivity indexes of these three parameters are listed in TABLE VII.

\section{A. Effects of coating materials}

The coating material of cases 1 to 8 is acrylate while it is polyimide for cases 9 to 16 . Although polyimide coating is thinner and stiffer than acrylate, the strain transfer fraction with polyimide coating is only a few percents better than the acrylate counterparts in all cases. This phenomenon is supported by the negligible values of the first-order and total sensitivity indexes of the radius and shear modulus of polymeric coating. According to the first-order and total sensitivity indexes, in
TABLE VI

CHANGE OF STRAIN TRANSFER FRACTION BY THE VARIATIONS OF THE GEOMETRICAL AND MATERIAL PROPERTIES OF OPTICAL FIBRE

\begin{tabular}{lll} 
Case & $\begin{array}{l}\text { Sum of } S_{i} \text { for } r_{f}, r_{c}, \\
E_{f}, G_{c} \text { and } G_{a}\end{array}$ & $\begin{array}{l}\Delta \beta \text { by } r_{f}, r_{c}, E_{f}, G_{c} \\
\text { and } G_{a}\end{array}$ \\
\hline 1 & 0.004 & $0.16 \%$ \\
2 & 0.043 & $0.99 \%$ \\
3 & 0.005 & $0.22 \%$ \\
4 & 0.007 & $0.25 \%$ \\
\hline 5 & 0.047 & $0.03 \%$ \\
6 & 0.092 & $0.73 \%$ \\
7 & 0.095 & $0.45 \%$ \\
8 & 0.114 & $0.32 \%$ \\
\hline 9 & 0.002 & $0.09 \%$ \\
10 & 0.021 & $0.45 \%$ \\
11 & 0.003 & $0.12 \%$ \\
12 & 0.004 & $0.15 \%$ \\
\hline 13 & 0.048 & $0.01 \%$ \\
14 & 0.034 & $0.26 \%$ \\
15 & 0.038 & $0.14 \%$ \\
16 & 0.043 & $0.10 \%$
\end{tabular}

TABLE VII

LIST OF THE FIRST-ORDER AND TOTAL SENSITIVITY INDEXES

\begin{tabular}{c|cc|cc|cc} 
& \multicolumn{2}{|c}{$t$} & \multicolumn{2}{c}{$L$} & \multicolumn{2}{c}{$\Delta_{z}$} \\
\hline Case & $S_{i}$ & $S_{T i}$ & $S_{i}$ & $S_{T i}$ & $S_{i}$ & $S_{T i}$ \\
\hline 1 & 0.016 & 0.028 & 0.736 & 0.832 & 0.150 & 0.231 \\
2 & 0.264 & 0.277 & 0.672 & 0.691 & - & - \\
3 & 0.022 & 0.035 & 0.700 & 0.823 & 0.151 & 0.261 \\
4 & 0.030 & 0.045 & 0.712 & 0.848 & 0.099 & 0.200 \\
\hline 5 & 0.160 & 0.413 & 0.315 & 0.673 & 0.092 & 0.323 \\
6 & 0.562 & 0.572 & 0.333 & 0.342 & - & - \\
7 & 0.592 & 0.618 & 0.107 & 0.164 & 0.132 & 0.191 \\
8 & 0.674 & 0.702 & 0.001 & 0.030 & 0.137 & 0.193 \\
\hline 9 & 0.021 & 0.038 & 0.687 & 0.814 & 0.163 & 0.273 \\
10 & 0.457 & 0.478 & 0.495 & 0.520 & - & - \\
11 & 0.030 & 0.047 & 0.658 & 0.803 & 0.158 & 0.304 \\
12 & 0.041 & 0.061 & 0.658 & 0.823 & 0.114 & 0.243 \\
\hline 13 & 0.183 & 0.585 & 0.209 & 0.665 & 0.067 & 0.356 \\
14 & 0.759 & 0.771 & 0.193 & 0.202 & - & - \\
15 & 0.762 & 0.793 & 0.059 & 0.100 & 0.083 & 0.122 \\
16 & 0.824 & 0.859 & 0.000 & 0.020 & 0.074 & 0.112
\end{tabular}

general, sensors with acrylate coating are more sensitive to bond length, while the sensors with polyimide coating are more sensitive to the thickness of adhesive beneath the optical fibre. It is because the shear modulus of acrylate coating is much lower than adhesive, the shear deformation is mainly on the acrylate coating layer, and hence it is less sensitive to the thickness of adhesive beneath the optical fibre.

\section{B. Effects of bond length}

The bond length of cases 1 to 4 and 9 to 12 is short $(15 \mathrm{~mm})$ while it is long $(30 \mathrm{~mm})$ for cases 5-8 and 13-16. For long bond length, the strain transfer fractions are close to hundred percent in most cases except the integral type strain sensor. If the structure allows sufficient bond length, long gauge length should be chosen. Hence, the uncertainties due to variation of workmanship can be minimised.

However, when OFBG strain sensor is used to transform other physical or chemical quantities to strain while the bond length is limited for miniature of sensor or other constraints, short bond length is the only choice (as in the case of 3D strain sensor in [10]). For short bond length, even under uniform strain field, the strain transfer factor varies from about $60 \%$ to 
$100 \%$. The most sensitive parameters for short gauge length is the bond length, which contributes about $70 \%$ of variation alone and more than $80 \%$ with the combined effects with other parameters. Hence, to minimise the variation of the strain transfer fraction of OFBG strain sensor with short bond length, the most efficient way is to control the variation of the bond length. The second most sensitive parameter for short bond length cases is the deviation of the grating position from the midpoint of the bond length, which contributes about $15 \%$ of variation alone and about $30 \%$ of variation with the combined effects with other parameters. On the other hand, the strain transfer fraction of short bond length OFBG strain sensor is insensitive to the thickness of adhesive beneath the optical fibre.

\section{Effects of strain field}

For uniform strain field, the difference between OFBG and integral strain sensor will be discussed in Section IV-D. In this section, only the OFBG strain sensor is discussed. Cases 1, 5, 9 and 13 are OFBG strain sensors under uniform strain field. For long bond length, the strain transfer fraction is close to hundred percent. For short bond length, the strain transfer fraction varies between about $55 \%$ and $100 \%$. From the firstorder sensitivity indexes, for short bond length, the bond length and the deviation of the grating from the mid-point of the bond length contributes about $70 \%$ and $15 \%$, respectively of variation. From the total sensitivity indexes, about $10 \%$ of additional variation is contributed by combined effects with other parameters.

Cases 3, 7, 11 and 15 are the OFBG sensors under quadratic strain field. For long bond length, the strain transfer fraction is higher than $95 \%$. The major sensitive factor is the thickness of adhesive beneath the optical fibre, which contributes about $60 \%$ and $76 \%$ for acrylate coated and polyimide coated optical fibre, respectively. For short bond length, the strain transfer fraction varies between about $50 \%$ and $100 \%$. The most sensitive factor is the bond length, which contributes about $70 \%$ of variation alone and $10 \%$ additional variation with effects of other parameters. While the strain transfer fraction is insensitive to the thickness of adhesive beneath the optical fibre, the deviation of the grating from the mid-point of the bond length contributes about $15 \%$ alone and 30\% with combined effects of other parameters.

Cases 4, 8, 12 and 16 are the OFBG strain sensors under reciprocal strain field. For long bond length, the strain transfer fraction is more than $96 \%$. For short bond length, the strain transfer fraction varies between $62 \%$ and $100 \%$. The variation of bond length contributes about $65 \%$ variation alone and $15 \%$ more with combination of other parameters. With short bond length under reciprocal strain field, the strain field in fibre may not be able to capture the high strain gradient near the stress concentration region (the double peaks in Fig. 7). With noise of the measured strain the optical fibre, it is difficult to reconstruct the original strain field.

\section{Sensor types}

The cases 2, 6, 10 and 14 are integral type strain sensors while all others are OFBG strain sensors with $10 \mathrm{~mm}$ grating.
Since all OFBG sensors have been discussed, only the integral strain sensor under uniform strain field will be discussed in this section. For integral strain sensor with long bond length, the strain transfer fraction varies from about $90 \%$ and $98 \%$, the range of which is larger than OFBG strain sensor with long bond length. The most sensitive parameter is the thickness of adhesive beneath the optical fibre. However, it contributes more than $75 \%$ of variation for polyimide coated sensor while less than $60 \%$ for acrylate coated sensor. On the other hand, the variation of bond length contributes more than $30 \%$ of acrylate coated sensor while it is about $20 \%$ of polyimide coated sensor. For short bond length, the strain transfer fraction varies between $70 \%$ and $95 \%$. Unlike the short bond length OFBG cases as well as short bond length integral sensor with acrylate coating, for which the most sensitive parameter is bond length, the sensitivity of the thickness of adhesive beneath the optical fibre of polyimide coated sensor is approximately the same as the bond length. It can be explained by the relatively low shear modulus of acrylate coating compared to adhesive, so the shear deformation is mainly in the coating instead of the adhesive layer.

\section{Conclusions}

In this paper, the first-order and total sensitivity indexes on the strain transfer fraction based on a well-established semianalytic/empirical model from the typical ranges of three material and five geometrical properties of common configuration of surface attached optical fibre strain sensor were investigated. Based on the quantitative global sensitivity analysis, it was found that all the most critical parameters, which are (i) the thickness of adhesive beneath the optical fibre, (ii) the bond length and (iii) the deviation of the grating from the mid-point of the bond length, are related to the workmanship instead of the properties of materials used.

The strain transfer fraction of the polyimide coated sensors are always a few percent higher than the counterparts of acrylate coated sensors. For polyimide coated sensor, the thickness of adhesive beneath the optical fibre is the most critical parameter while it is the bond length for those acrylate coated sensors. If the situation is permissible, long bond length can reduce the variation due to workmanship and achieve much less variation of strain transfer fraction of OFBG strain sensors under various types of strain field. Under non-uniform strain field, the deviation of the grating from the mid-point should be controlled, especially when strain gradient is high as the reciprocal strain field in this study. For integral strain sensor, the variation due to the thickness beneath the optical fibre is comparable to the bond length.

\section{ACKNOWLEDGEMENT}

The authors would like to thank the support of Prof Christopher K.Y. Leung from the Department of Civil and Environmental Engineering of the Hong Kong University of Science and Technology. 


\section{REFERENCES}

[1] S. Ezekiel, "Fiber optic gyros," in Proceedings of SPIE 15th Anniversary Conference, 1992.

[2] S. F. Masri, M. S. Agbabian, A. M. Abdel-Ghaffar, M. Higazy, R. O. Claus, and M. J. de Vries, "Experimental study of embedded fiber-optic strain gauges in concrete structures," Journal of Engineering Mechanics, vol. 120, no. 8, pp. 1696-1717, 1994.

[3] W. Habel and D. Hofmann, "Strain measurements in reinforced concrete walls during the hydration reaction by means of embedded fiber interformeters," in Proceedings of 2nd European Conference on Smart Structures and Materials, SPIE, vol. 2361, 1994, pp. 176-179.

[4] D. Inaudi, "Coherence multiplexing of in-line displacement and temperature sensors," Optical Engineering, vol. 34, no. 7, pp. 1912-1915, 1995.

[5] K. O. Hill and G. Meltz, "Fiber Bragg grating technology fundamentals and overview," Journal of Lightwave Technology, vol. 15, no. 8, pp. 1263-1276, 1997.

[6] Y. Rao, "In-fibre Bragg grating sensors," Measurement Science and Technology, vol. 8, no. 4, pp. 355-375, 1997.

[7] A. D. Kersey, M. A. Davis, H. J. Patrick, M. LeBlanc, K. P. Koo, C. G. Askins, M. A. Putnam, and E. J. Friebele, "Fiber grating sensors," Journal of Lightwave Technology, vol. 15, no. 8, pp. 1442-1462, 1997.

[8] E. Mehrani, A. Ayoub, and A. Ayoub, "Evaluation of fiber optic sensors for remote health monitoring of bridge structures," Materials and Structures/Materiaux et Constructions, vol. 42, no. 2, pp. 183-199, 2009.

[9] Y. Kim, H. Sung, H. Kim, and J. Kim, "Monitoring of tension force and load transfer of ground anchor by using optical FBG sensors embedded tendon," Smart Structures and Systems, vol. 7, no. 4, 2011.

[10] Z. Zhou, W. Liu, Y. Huang, H. Wang, H. Jianping, M. Huang, and O. Jinping, "Optical fiber Bragg grating sensor assembly for 3D strain monitoring and its case study in highway pavement," Mechanical Systems and Signal Processing, vol. 28, pp. 36-49, 2012.

[11] Y. Jiang, Y. Xu, and C. K. Leung, " $3 * 3$ coupler-based mach-zehnder interferometer and its application in the delamination detection in frp composite," Journal of Intelligent Material Systems and Structures, vol. 19 , pp. 497-507, 2008.

[12] A. Kerrouche, W. J. O. Boyle, T. Sun, K. T. V. Grattan, J. Schmidt, and B. Taljsten, "Strain measurement using embedded fiber Bragg grating sensors inside an anchored carbon fiber polymer reinforcement prestressing rod for structural monitoring," Sensors Journal, IEEE, vol. 9, no. 11, pp. 1456-1461, 2009.

[13] S. Yashiro and T. Okabe, "Estimation of fatigue damage in holed composite laminates using an embedded FBG sensor," Composites: Part A, vol. 42, pp. 1962-1969, 2011.

[14] J. L. Otegui, U. H. Mohaupt, and D. J. Burns, "A strain gauge technique for monitoring small fatigue cracks in welds," Engineering Fracture Mechanics, vol. 40, no. 3, pp. 549-569, 1991.

[15] H. . Zhu, J. . Yin, J. . Dong, and L. Zhang, "Physical modelling of sliding failure of concrete gravity dam under overloading condition," Geomechanics and Engineering, vol. 2, no. 2, pp. 89-106, 2010.

[16] Y. Wang, L. Woodworth, and B. Han, "Simultaneous measurement of effective chemical shrinkage and modulus evolutions during polymerization," Experimental Mechanics, vol. 51, no. 7, pp. 1155-1169, 2011.

[17] T. Yeo, T. Sun, K. Grattan, D. Parry, R. Lade, and B. Powell, "Characterisation of a polymer-coated fibre Bragg grating sensor for relative humidity sensing," Sensors and Actuators B: Chemical, vol. 110, pp. $148-155,2005$.

[18] H. Zhu, A. N. L. Ho, J. Yin, H. W. Sun, H. Pei, and C. Hong, "An optical fibre monitoring system for evaluating the performance of a soil nailed slope," Smart Structures and Systems, vol. 9, no. 5, pp. 393-410, 2012.

[19] H. Pei, J. Yin, H. Zhu, C. Hong, W. Jin, and D. Xu, "Monitoring of lateral displacements of a slope using a series of special fibre Bragg grating-based in-place inclinometers," Measurement Science and Technology, vol. 23, no. 2, 2012.

[20] Y. Q. Ni, Y. Xia, W. Y. Liao, and J. M. Ko, "Technology innovation in developing the structural health monitoring system for guangzhou new tv tower," Structural Control and Health Monitoring, vol. 16, no. 1, pp. 73-98, 2009.

[21] M. R. Mokhtar, K. Owens, J. Kwasny, S. E. Taylor, P. A. M. Basheer, D. Cleland, Y. Bai, M. Sonebi, G. Davis, A. Gupta, I. Hogg, B. Bell, W. Doherty, S. McKeague, D. Moore, K. Greeves, T. Sun, and K. T. V. Grattan, "Fiber-optic strain sensor system with temperature compensation for arch bridge condition monitoring," IEEE Sensors Journal, vol. 12 , no. 5 , pp. $1470-1476,2012$.
[22] F. Ansari and Y. Libo, "Mechanics of bond and interface shear transfer in optical fiber sensors," Journal of Engineering Mechanics, vol. 124, no. 4, pp. 385-394, 1998

[23] G. Duck, G. Renaud, and R. Measures, "Mechanical load transfer into a distributed optical fiber sensor due to a linear strain gradient: Embedded and surface bonded cases," Smart Materials and Structures, vol. 8, no. 2 , pp. 175-181, 1999

[24] D. Li, H. Li, L. Ren, and G. Song, "Strain transferring analysis of fiber Bragg grating sensors," Optical Engineering, vol. 45, no. 2, 2006.

[25] H. Li, G. Zhou, L. Ren, and D. Li, "Strain transfer analysis of embedded fiber Bragg grating sensor under nonaxial stress," Optical Engineering, vol. 46, no. 5, 2007.

[26] K. T. Wan, C. K. Y. Leung, and N. G. Olson, "Investigation of the strain transfer for surface-attached optical fiber strain sensors," Smart Materials and Structures, vol. 17, no. 3, 2008.

[27] S. . Her and C. . Huang, "Effect of coating on the strain transfer of optical fiber sensors," Sensors, vol. 11, no. 7, pp. 6926-6941, 2011.

[28] V. Schukar, N. Kusche, and W. Habel, "How reliably do fiber Bragg grating patches perform as strain sensors?" Sensors Journal, IEEE, vol. 12 , no. 1, pp. 128-132, 2012.

[29] H. L. Cox, "The elasticity and strength of paper and other fibrous materials," British Journal of Applied Physics, vol. 3, no. 3, pp. 72 79, 1952.

[30] T. Turanyi, "Sensitivity analysis of complex kinetic systems. tools and applications," Journal of Mathematical Chemistry, vol. 5, pp. 203-248, 1990.

[31] J. D. Cawlfield and M.-C. Wu, "Probabilistic sensitivity analysis for one-dimensional reactive transport in porous media," Water Resources Research, vol. 29, no. 3, pp. 661-672, 1993

[32] I. M. Sobol, "Sensitivity estimates for nonlinear mathematical models," Mathematical Modelling and Computational Experiments, vol. 1, no. 4, pp. 407-414, 1993.

[33] A. Saltelli, T. H. Andres, and T. Homma, "Sensitivity analysis of model output. an investigation of new techniques," Computational Statistics and Data Analysis, vol. 15, no. 2, pp. 211-238, 1993.

[34] R. I. Cukier, C. M. Fortuin, K. E. Shuler, A. G. Petschek, and J. H. Schaibly, "Study of the sensitivity of coupled reaction systems to uncertainties in rate coefficients. i theory," The Journal of chemical physics, pp. 3873-3878, 1970.

[35] A. Saltelli and R. Bolado, "An alternative way to compute fourier amplitude sensitivity test (fast)," Computational Statistics and Data Analysis, vol. 26, no. 4, pp. 445-460, 1998.

[36] A. Saltelli, S. Tarantola, and K. P.-S. Chan, "A quantitative modelindependent method for global sensitivity analysis of model output," Technometrics, vol. 41, no. 1, pp. 39-56, 1999.

[37] K. Peters, M. Studer, J. Botsis, A. Iocco, H. Limberger, and R. Salathe, "Embedded optical fiber Bragg grating sensor in a nonuniform strain field: Measurements and simulations," Experimental Mechanics, vol. 41, no. 1, pp. 19-28, 2001

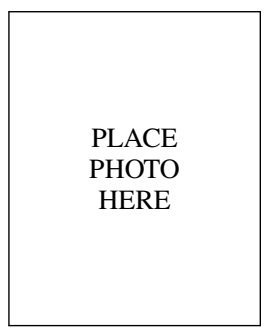

Kai Tai Wan Dr K.T. Wan is currently technical manager of the working group in Construction and Building Materials of the Research and Development Division of Nano and Advanced Materials Institute Limited, Hong Kong. He received his Ph.D. in Civil Engineering from the Hong Kong University of Science and Technology in 2005. His areas of expertise include advanced sensory technologies for infrastructures, structural health monitoring, cementitious materials and structural strengthening/retrofitting. $\mathrm{He}$ has developed various kinds of optical fibre sensor for infrastructure that included a distributed crack sensor, a corrosion sensor, a delamination sensor, a vibration sensor, an integral strain sensor and a distributed pressure sensor. He is the executive board member of Hong Kong Concrete Institute, director of the Board of Direction of American Society of Civil Engineers Hong Kong Section and members of various technical committees of International Union of Laboratories and Experts in Construction Materials, Systems and Structures. 